\title{
Video Article \\ A Novel Three-dimensional Flow Chamber Device to Study Chemokine- directed Extravasation of Cells Circulating under Physiological Flow Conditions
}

\author{
Valentina Goncharova ${ }^{1}$, Sophia K. Khaldoyanidi ${ }^{1,2}$ \\ ${ }^{1}$ Torrey Pines Institute for Molecular Studies \\ ${ }^{2}$ Cascade LifeSciences Inc.
}

Correspondence to: Sophia K. Khaldoyanidi at skhaldoyanidi@tpims.org

URL: https://www.jove.com/video/50959

DOI: doi:10.3791/50959

Keywords: Bioengineering, Issue 77, Cellular Biology, Biophysics, Physiology, Molecular Biology, Biomedical Engineering, Immunology, Cells, Biological Factors, Equipment and Supplies, Cell Physiological Phenomena, Natural Science Disciplines, Life Sciences (General), circulating cells, extravasation, physiological shear stress, endothelial cells, microenvironment, chemokine gradient, flow, chamber, cell culture, assay

Date Published: 7/15/2013

Citation: Goncharova, V., Khaldoyanidi, S.K. A Novel Three-dimensional Flow Chamber Device to Study Chemokine-directed Extravasation of Cells Circulating under Physiological Flow Conditions. J. Vis. Exp. (77), e50959, doi:10.3791/50959 (2013).

\section{Abstract}

Extravasation of circulating cells from the bloodstream plays a central role in many physiological and pathophysiological processes, including stem cell homing and tumor metastasis. The three-dimensional flow chamber device (hereafter the 3D device) is a novel in vitro technology that recreates physiological shear stress and allows each step of the cell extravasation cascade to be quantified. The 3D device consists of an upper compartment in which the cells of interest circulate under shear stress, and a lower compartment of static wells that contain the chemoattractants of interest. The two compartments are separated by porous inserts coated with a monolayer of endothelial cells (EC). An optional second insert with microenvironmental cells of interest can be placed immediately beneath the $\mathrm{EC}$ layer. A gas exchange unit allows the optimal $\mathrm{CO}_{2}$ tension to be maintained and provides an access point to add or withdraw cells or compounds during the experiment. The test cells circulate in the upper compartment at the desired shear stress (flow rate) controlled by a peristaltic pump. At the end of the experiment, the circulating and migrated cells are collected for further analyses. The 3D device can be used to examine cell rolling on and adhesion to EC under shear stress, transmigration in response to chemokine gradients, resistance to shear stress, cluster formation, and cell survival. In addition, the optional second insert allows the effects of crosstalk between EC and microenvironmental cells to be examined. The translational applications of the 3D device include testing of drug candidates that target cell migration and predicting the in vivo behavior of cells after intravenous injection. Thus, the novel 3D device is a versatile and inexpensive tool to study the molecular mechanisms that mediate cellular extravasation.

\section{Video Link}

The video component of this article can be found at https://www.jove.com/video/50959/

\section{Introduction}

Cell extravasation is the process by which circulating cells exit the bloodstream and is a critical component of many physiological responses in the body. The process is also important for tissue regeneration, as for example, when therapeutic cells mobilized from tissues into the blood vessels (or injected intravenously) migrate through the vasculature and exit the circulation to sites of injury or degeneration. Extravasation is also a critical component of the pathogenesis of many diseases, including inflammation, immune rejection, autoimmunity, and tumor metastasis.

Extravasation is a complex multi-step process that involves the interaction of circulating cells with endothelial cells (EC) under conditions of physiological flow. The process comprises (a) cell rolling, (b) firm adhesion to the luminal surface of EC, and (c) transmigration across the EC Importantly, each step of the extravasation cascade is regulated by a subset of cell type-specific and species-specific molecules. However, the detailed molecular mechanisms that regulate extravasation of specific cell subsets are not well understood, largely due to the technical difficulty of recapitulating the conditions in the bloodstream. The novel 3D device described here is designed to overcome these technical challenges and allow experiments to be performed that will improve our understanding of the biology of cell migration.

The molecules that mediate cell migration are important therapeutic targets. A detailed understanding of the molecular events that control the migration of specific cell types will assist in identifying novel targets for the therapeutic promotion or inhibition of extravasation. For example, interventions that enhance the migration of therapeutic stem cells (whether derived from adult, neonatal or even from fetal tissues) toward sites of injury or degeneration would be of great utility in tissue regeneration. There is growing interest in the in vitro generation of therapeutic stem cells, including cells derived from pluripotent sources, and in ex vivo manipulated adult stem cells (expanded, genetically manipulated, and pretreated with various enzymes) ${ }^{1}$. Thus, there is a need for novel technologies to evaluate the quality of the stem cells before they are used for therapeutic purposes. Among other parameters, the cells must be able to efficiently exit the circulation, because treatments for multifocal disorders may require intravenous administration of the stem cells ${ }^{2}$. Indeed, one of the current challenges in stem cell biology is to overcome the extremely low efficiency with which stem cells home to sites of tissue damage ${ }^{3-6}$, highlighting the need to address this gap in our understanding 
of stem cell migration. In contrast, strategies that block cell migration by targeting specific homing molecules would be useful for the treatment of inflammatory and autoimmune diseases as well as metastatic cancer. Thus, understanding the molecular mechanisms that mediate the interactions between circulating cells and EC during cell migration and extravasation is relevant to translational medicine and drug discovery as well as to basic science.

There are currently a number of methods available to study different aspects of cell migration. However, these methods have shortcomings that can be overcome with the new 3D device.

\section{Animal models:}

Animal models, such as immunocompromised mice and genetically manipulated mice, have been useful tools to study the migration of human cells in vivo. However, one significant drawback to these models is that human cells interact poorly with adhesion molecules present on mouse EC, in part due to species-specific sequence differences in many cell surface molecules. Thus, the use of rodents to study human cell migration is unlikely to authentically reflect the events in human organ-specific vascular beds. In addition, in vivo models are not suitable for highthroughput screening of drug candidates. The conventional in vivo models using to study cell homing do not discriminate between the different steps of the extravasation cascade, making it difficult to identify and target novel homing molecules. The intravital microscopy approach was developed to address this need and has been informative; however, this technique is extremely time- and labor-intensive ${ }^{7,8}$.

\section{Static transmigration assays:}

Transwell or Boyden chamber assays measure cell migration across a porous membrane and are widely used in migration studies. The assay has the advantage that it can be used to study not only cell motility and cell-extracellular matrix (ECM) interactions, but also chemokine-mediated migration of cells across an EC monolayer grown on the porous membranes. Unfortunately, the phenotype and function of EC under static conditions differ significantly from those under physiological flow ${ }^{9,10}$. Thus, the chemotactic events that occur in Transwell assays do not faithfully mimic the interactions between migrating cells and EC under shear stress. In addition, the "rolling" step of the homing cascade, which adds an extra dimension of selectivity to the overall process, does not occur in static Transwell assays. Thus, while this technology does allow quantitative evaluation of chemokine-mediated cell migration across the EC monolayer, it is limited by its inability to provide the shear stress that mimics blood flow in vivo.

Assays under shear stress:

Wall shear stress is known to play an important role in regulating EC function ${ }^{9,10}$. Shear forces induce rapid activation of signaling cascades, transcription factors, and differential gene expression in EC ${ }^{11,12}$. This information led to the development of the next generation of adhesion assays - parallel laminar flow chambers and capillaries - to study rolling and adhesion of cells to EC under conditions of shear stress ${ }^{7,13}$. The limiting factor for these assays is that they can measure only rolling and adhesion, but do not differentiate between an adherent cell that would go on to transmigrate and an adherent cell that is "arrested" on the EC monolayer and would not transmigrate. Moreover, these assays cannot measure migration of cells toward a chemokine gradient.

Several reports have described the ability of adherent cells to crawl beneath an EC monolayer grown on glass slides under conditions of flow 14. The limitations to this crawling technique include: it analyzes only a limited number of cells; it is non-quantitative; it analyzes cell crawling on the matrix and cell surface but not migration toward a chemotactic gradient; and it does not allow the transmigrated cells to be isolated. Thus, although this assay has the advantage of applying shear stress to the EC monolayer, it cannot quantify migration of cells toward a chemokine gradient.

The novel 3D technology described here overcomes many of these shortcomings by combining shear force (upper compartment) with a static chemokine gradient (lower compartment) and permits quantitative evaluation of each step of the extravasation cascade (Figure 1). The device can also be used to investigate how crosstalk between EC and the microenvironment influences the ability of EC to support extravasation of circulating cells. The following protocol describes the step-by-step methodology for using the 3D flow chamber device.

\section{Protocol}

\section{Prepare the Upper and Lower Inserts for Cell Growth by Collagen Coating}

1. Calculate the number of inserts needed for the experiment. Place each insert in a separate sterile Petri dish ( $35 \times 10 \mathrm{~mm})$ and sterilize by irradiation (11 Gy).

2. Prepare the collagen solution for coating of membranes: $50 \mu \mathrm{g} / \mathrm{ml}, 154 \mu \mathrm{l}$ per insert (insert surface area $1.54 \mathrm{~cm}^{2}$ ).

3. Place several drops $(\sim 20 \mu \mathrm{l}$ per drop) of collagen solution in a circle on the surface of the insert membrane (do not let the pipette tip touch the surface) and then carefully add the rest of the aliquot to form one large drop on the membrane surface. Ensure that the collagen solution remains hemispherical and does not drain from the membrane surface.

4. Cover the Petri dish with a lid and incubate for $1 \mathrm{hr}$ at room temperature on a flat surface.

5. Aspirate the collagen solution and wash the membrane once by covering with $160 \mu \mathrm{IPBS}$.

6. Aspirate the PBS and replace the lid. If using the same day, place the Petri dish in a dry place at room temperature. Otherwise, place the dish at $4{ }^{\circ} \mathrm{C}$ and use the insert within 7 days.

\section{Culture Endothelial Cells on the Upper Inserts}

1. Prepare EC suspension in desired culture medium. Ensure that the cell viability is $>98 \%$ and use immediately. 
Note: For human umbilical vein EC (HUVEC), 4 × $10^{5}$ cells in $160 \mu \mathrm{l}$ per insert is used.

2. Take the Petri dishes with collagen-coated inserts prepared in section 1; do not remove the inserts from the dishes. Place several small drops of the cell suspension (approximately $20 \mu \mathrm{l}$ per drop) in a circle on the surface of the collagen-coated membrane (do not let the pipette tip touch the surface) and then carefully add the rest of the cell suspension to form one large drop. Ensure that the cell suspension remains hemispherical and does not drain from the membrane surface.

3. Replace the lids and carefully place the dishes in a $5 \% \mathrm{CO}_{2}$ cell culture incubator and incubate (without shaking) at $37{ }^{\circ} \mathrm{C}$ for $30 \mathrm{~min}$ to allow the cells to attach to the membrane.

4. Gently add $5 \mathrm{ml}$ of culture medium to each Petri dish ensuring that the insert remains on the bottom of the dish and is completely covered by the medium. Replace the lids and carefully put the dishes back in the incubator. Culture the cells at $37^{\circ} \mathrm{C}$ overnight.

5. Use the same approach to prepare the second (optional) lower insert with cells representing the local microenvironment, which will be placed below the insert containing the EC layer.

Note: if the lower inserts are included in the experiments, the upper and lower inserts are first connected to each other before placing the inserts into the 3D device (Figure 4B).

\section{Assemble the 3D Flow Chamber Device}

1. Screw together the upper and lower plates, connect the plates to the gas exchange unit using tubing, place the assembled device into a clean plastic bag, and sterilize the bag and contents by irradiation (11 Gy).

2. Remove a tray-shelf from the cell culture incubator, place into a sterile tissue culture hood, spray with $70 \%$ ethanol, wipe off, and then cover the tray with a large sterile napkin.

3. Place the irradiated plastic bag into the hood, remove the device from the bag and place on the sterile incubator tray. Connect the device to the peristaltic pump with the tubing provided. Program the pump for an optimal speed of $0.2 \mathrm{ml} / \mathrm{min}$.

Note: To connect the pump with the electrical socket, extrude the electrical cord through the hole in the backside of the incubator. Use robber plug around the cord to assure that the hole is airtight.

4. Place $5-7 \mathrm{ml}$ of desired culture medium into a sterile $15 \mathrm{ml}$ tube (for the "inlet").

\section{Prime the 3D Flow Chamber Device}

1. Disconnect the inlet tubing from the outlet by pulling the metal needle from the tubing (use small sterile napkins to maintain sterility of the tubing). Use the metal needle connected to the tubing as an "inlet" and the disconnected end of tubing as an "outlet". Place the metal needle inlet into the $15 \mathrm{ml}$ tube with the media; place the outlet into the empty $15 \mathrm{ml}$ tube.

2. Turn on the pump so the flow is counterclockwise and set the flow rate at $0.2 \mathrm{ml} / \mathrm{min}$. Allow the negative pressure to draw the medium from the $15 \mathrm{ml}$ inlet tube into the tubing and stop the pump when the medium reaches the end of tubing immediately before it connects to the device (Figure 2, Stop \#1).

3. Unscrew the upper and lower plates of the device. Carefully remove the upper plate and place 1,500 - 1,550 $\mu$ l of medium (either medium alone or plus the experimental chemokines) into the lower wells. Make sure the well contains sufficient medium to form a hemisphere that reaches approximately $1-2 \mathrm{~mm}$ above the lower plate.

4. Transfer the prepared insert from the Petri dish to the wells of the lower plate using sterile forceps, making sure that no bubbles are trapped beneath the inserts. Aspirate any medium that appears on the surface of the lower plate to ensure that the plate remains dry. Place the upper plate back onto the lower plate and re-connect the plates using the screws.

5. Immediately turn on the peristaltic pump and allow medium to flow through the 3D device. Elevate the outlet end of the device and maintain the chamber in this position to prevent formation of bubbles within the space between the plates.

6. Allow the medium to fill the chamber and the tubing connecting the chamber to the gas exchange unit. Stop the pump immediately before the medium reaches the gas exchange unit (Figure 2; Stop \#2). Place $3 \mathrm{ml}$ of medium into the gas exchange unit, turn on the pump, and allow the air bubbles to escape through the gas exchange unit. Elevate the gas exchange unit to allow the medium to fill the tubing exiting the gas exchange unit and stop the pump when the medium reaches $2-3 \mathrm{~cm}$ before the end of the outlet tubing (Figure 2, Stop \#3).

7. Connect the inlet metal needle to the outlet using small sterile napkins. Reprogram the pump so the medium flows in the opposite direction (clockwise) toward the gas exchange unit and ensure any air bubbles are removed once they reach the gas exchange unit. Check that no air bubbles remain in the system.

8. Reprogram the pump so the medium flows counterclockwise. Add $100 \mu \mathrm{l}$ of the test cell suspension to the gas exchange unit. Close the lid of the gas exchange unit, place the tray with the working system back into the incubator, and allow the test cells to circulate in the 3D device at $37^{\circ} \mathrm{C}$ for the desired time.

Note: Clean the electrical cord of the pump with $70 \%$ ethanol and place it inside of the incubator.

\section{Terminate the Test and Collect Samples for Assay}

1. Remove the tray with the working system from the incubator and place in the hood. Stop the pump, disconnect the inlet and outlet, and place the ends into empty sterile $15 \mathrm{ml}$ tubes.

2. Turn on the pump and collect the cell suspension from the system; place the cell suspension on ice, if appropriate, until use.

3. Disassemble the chamber by removing the screws and lifting off the upper plate. Remove the inserts from the lower plate and transfer them to Petri dishes. 
Note: The inserts can be stained to visualize and count cells in situ (crystal violet $0.05 \%$ in $\mathrm{dH}_{2} \mathrm{O}$ ) or trypsinized to collect the EC for further testing.

4. Remove the medium and cells from the lower wells into tubes and centrifuge for 5 min at $210 \times \mathrm{g}$. Remove the supernatant, wash and resuspend the cells as desired, and process the cells according to the specific experimental goals (discussed further below).

\section{Representative Results}

The murine bone marrow-derived EC line STR-12 was grown on inserts with $5 \mu \mathrm{m}$ pores. The rate of EC growth was monitored under a microscope and when the EC were $100 \%$ confluent, the inserts were transferred into the wells in the lower compartment of the 3D device. Immediately before placing the inserts, the wells of the lower compartment were filled with culture medium alone (negative control) or with medium supplemented with stromal cell-derived factor-1 (SDF-1; $5 \mathrm{ng} / \mathrm{ml}$ and $50 \mathrm{ng} / \mathrm{ml}$ ). Thereafter, the 3D device was assembled and the chamber was filled with medium as described in the protocol. The test cells to be circulated in the upper compartment of the device were freshly harvested murine bone marrow cells $\left(3.5 \times 10^{6}\right.$ cells per chamber). A defined shear stress of $0.8 \mathrm{dyn} / \mathrm{cm}^{2}$ was applied by setting the peristaltic pump speed at $0.2 \mathrm{ml} / \mathrm{min}$. The entire working system was then placed in the $5 \% \mathrm{CO}_{2}$ incubator at $37^{\circ} \mathrm{C}$ and the cells were allowed to circulate and interact with the EC monolayer for $4 \mathrm{hr}$. At the end of that time, the circulating cells were collected, the chamber was disassembled, and the inserts were removed as described in the protocol. The transmigrated cells were harvested from the lower wells, washed, resuspended in fresh medium, and transferred to methylcellulose cultures supplemented with hematopoietic growth factors for colony-forming cell (CFC) assay (Figure 3). As expected, we found a significantly higher number of CFC had migrated across the EC monolayer to the wells containing 50 ng/ml SDF-1 than to wells containing $5 \mathrm{ng} / \mathrm{ml}$ SDF- 1 or medium alone.

As we described earlier, none of the current in vitro techniques available to study cell migration are capable of testing the effect of the local microenvironment on the ability of EC to support extravasation of migrating cells. To illustrate how this can be achieved with the 3D device, we examined extravasation of circulating hematopoietic cells across a layer of EC and a layer of bone marrow stromal cells. For this, a second (lower) insert containing a layer of stromal cells was juxtaposed to the upper insert containing the EC monolayer in the lower plate (Figure 4). The experiment was then performed as described above and the transmigrated cells were harvested from the wells and counted. The results demonstrated that insertion of an additional layer of stromal cells beneath the EC monolayer significantly increased the migration of hematopoietic cells toward SDF-1 (Figure 4). This finding is consistent with the notion that the local microenvironment contributes to the recruitment of circulating cells to the tissue.

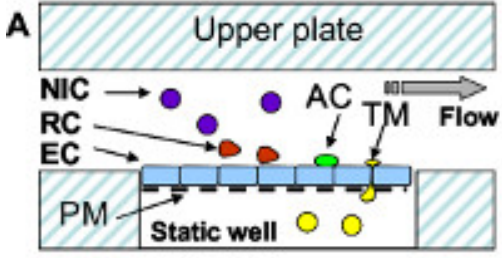

Lower plate
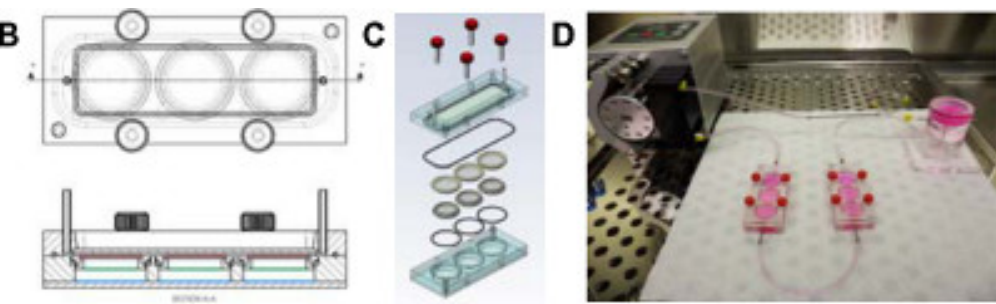

Figure 1. The 3D flow chamber device. A: The hermetically sealed 3D device consists of two compartments separated by a replaceable porous membrane (PM). EC are grown on the membrane, which then forms a barrier between the upper and lower compartments. Defined shear stresses are applied to the upper compartment of the 3D device by perfusing media using a constant infusion peristaltic pump. Circulating cells are either non-interacting (NIC), interact with the EC transiently ('roll'; RC), or adhere to the EC (AC). A subpopulation of adherent cells transmigrates across the EC layer to the lower static compartment of the device (TM). B: A top view drawing (upper panel) of the 3D device. The horizontal sectional view (lower panel) demonstrates the position of the upper membrane (red), the lower membrane (green), and the bottom of the well (blue). C: The isometric view illustrates the integral parts of the device: four screws, an acrylic top block, an upper chamber seal, upper and lower membranes, bottom o-ring, and a 3-well acrylic bottom block. D: A photograph of the 3D flow chamber connected to a peristaltic pump and a gas exchange unit. The system is assembled in a sterile hood and then transferred to a cell culture incubator. 


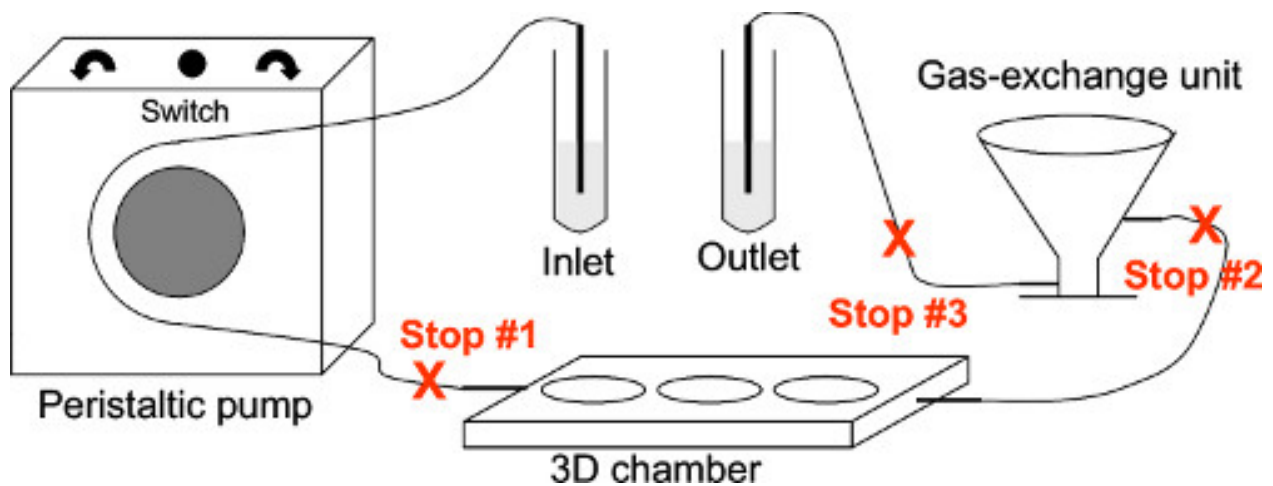

Figure 2. A schematic representation of the 3D device system. The drawing shows the three stops required to assemble the system. The culture medium is drawn in from the inlet by the negative pressure created by the peristaltic pump. The flow of medium is shut off by Stop \#1 to allow the inserts to be placed into the device. After the chamber is closed, the pump is switched on again and the chamber is immediately filled with medium to prevent drying of the cells grown on the inserts. Stop \#2 allows medium to be placed in the gas exchange unit. Stop \#3 allows the flow to be stopped for connection of the inlet and outlet. The flow can be directed in a clockwise or counterclockwise direction to remove air bubbles through the gas exchange unit by using a switch on the pump.

A

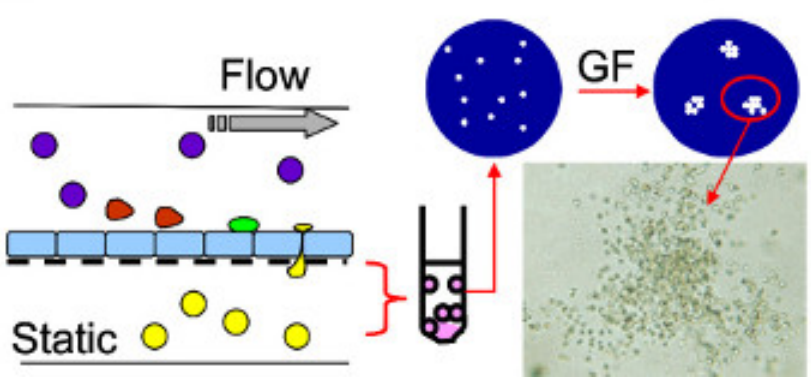

B

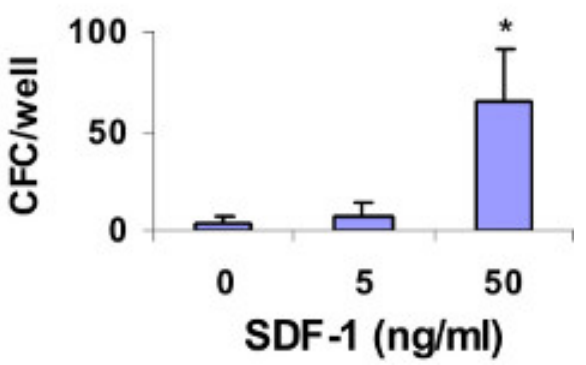

Figure 3. The 3D device supports SDF-1-mediated transmigration of hematopoietic progenitors under conditions of physiological shear stress. A: Three devices (each containing 3 wells in the lower compartment) were assembled and run in parallel. Medium alone or medium containing SDF-1 at 5 or $50 \mathrm{ng} / \mathrm{ml}$ was added to the lower compartment wells. Murine bone marrow cells were allowed to circulate in the devices for $4 \mathrm{hr}$. Thereafter, each device was disassembled and the cells that had transmigrated toward SDF-1 (yellow) were collected from the lower wells, counted, and cultured in methylcellulose supplemented with hematopoietic growth factors (GF). Fourteen days later, the colonies were scored under the microscope. B: The number of progenitors (colony-forming cells, CFC) recovered from the lower compartments is shown as mean \pm SD of three wells per condition. ${ }^{*} p<0.05$.
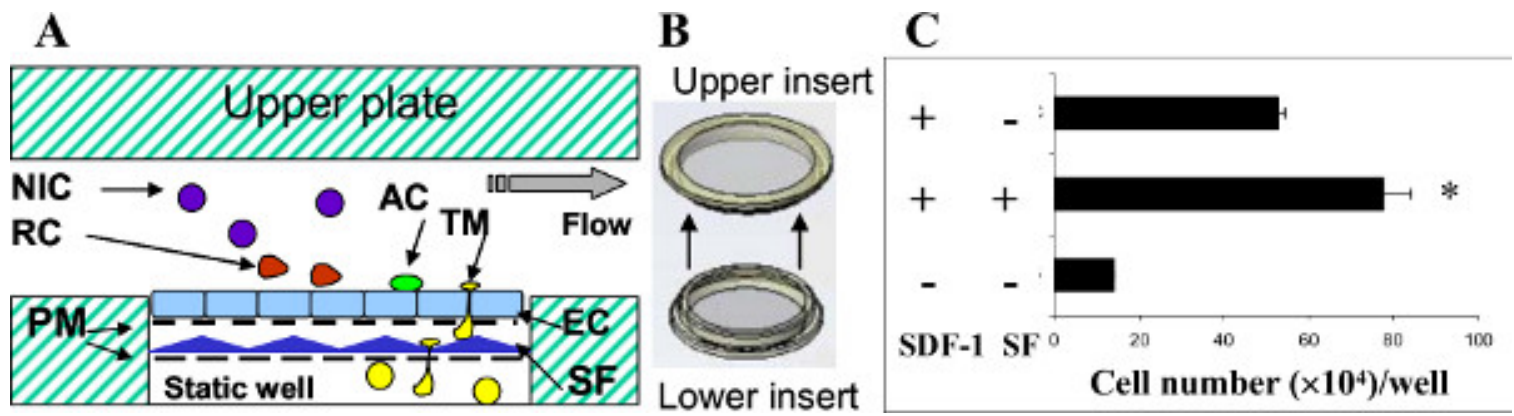

Figure 4. The effect of the microenvironment on interactions between hematopoietic cells and endothelial cells. A: The illustration shows the position of a second porous membrane with cultured stromal fibroblasts (SF) underneath the upper membrane with EC. Other abbreviations are as described for Figure 1A. B: The additional membrane (lower insert) with cultured SF was inserted beneath the upper membrane. C: Three devices (each containing 3 wells) were run in parallel. Medium alone or medium containing SDF-1 at $50 \mathrm{ng} / \mathrm{ml} \mathrm{SDF-1} \mathrm{was} \mathrm{added} \mathrm{to} \mathrm{the}$ lower compartment wells. The negative control chambers omitted SC, SDF-1, or both. Bone marrow cells were allowed to circulate for $4 \mathrm{hr}$ at 37 ${ }^{\circ} \mathrm{C}$. Thereafter, each device was disassembled and the transmigrated cells were collected from the lower compartment wells and counted. The number of transmigrated cells recovered is shown as the mean \pm SD of three wells per condition. ${ }^{*} p<0.05$.

\section{Discussion}

The 3D device can be a useful technology for research in various fields including stem cell biology, vascular biology, hematology, oncology, autoimmunity, and inflammation. The 3D device will be particularly useful for researchers studying hematopoietic, mesenchymal, neural, and other stem cells to investigate the molecular mechanisms regulating each step of the stem cell extravasation cascade. Researchers studying cell migration can also use this device to examine the differing migratory mechanisms of T- and B-lymphocytes, monocytes, neutrophils, eosinophils, NK cells, and other migratory cells. In vascular biology, the device will be useful for assessing the effect of the local microenvironment on the 
ability of EC to support cell recruitment and to mimic the blood-brain barrier in vitro. For researchers focusing on disease pathogenesis, the device can be used to study the migration of cytotoxic T cells into the pancreas during type I diabetes, the migration of eosinophils into the lungs of asthma patients, the migration of neutrophils, monocytes, and lymphocytes into sites of inflammation in a variety of disorders, the recruitment of cells to wound healing sites, the interaction of cytotoxic T cells with the neovasculature, and recruitment of cytotoxic T cells into tumors.

The novel 3D device will also be a useful tool for translational science and for preclinical drug development and screening. For example, the device can be used to optimize the preparation of therapeutic cell suspensions for intravenous administration, to test the recruitment of therapeutic cells to injured tissues versus normal tissues, and to examine the role of the specific organ or tissue endothelium and microenvironment in this process. For drug development, the device could be used to test drug candidates that target tumor cells and block each step of the extravasation cascade, to test migrating cells as a drug delivery vehicle to tumor sites, to test drugs that regulate the function of human organ-specific endothelium or the local tissue-specific microenvironment, and to test combinations of drugs that regulate different steps of the homing cascade. Finally, when converted into a high-throughput system, the device can be used for screening small molecules, peptides, and antibodies that target molecules mediating cell trafficking.

\section{Technical details and tips}

Rolling and adhesion under flow are the critical steps in the extravasation cascade and contribute significantly to the efficiency of cell migration in vivo. Notably, these steps do not contribute to static assays in vitro. However, static transmigration assays are useful as negative controls in experiments testing the effects of shear stress on cell survival and function, including the ability of EC to support low affinity interactions (rolling) and firm adhesion.

The choice of medium for experiments in the 3D device is important. Media contain different growth factors that may influence the survival of circulating cells, particularly during long incubation times. In addition, the concentrations of $\mathrm{Ca}^{2+}$ and $\mathrm{Mg}^{2+}$, which may influence adhesive interactions under conditions of physiological flow, vary considerably in commercial culture media. Other technical details that should be taken into account when planning experiments with the 3D device are discussed below.

\section{Selection of the EC monolayer}

The cell surface signature of EC is influenced by various parameters, including the species and tissue of origin and the cell culture conditions, which should be taken into account in the experimental design. Some commonly used EC include lung-derived microvascular EC (LDMVEC), bone marrow-derived EC (BMDEC), brain-derived EC (BDEC), and HUVEC. The properties of EC also vary with their origin. For example, BMDEC constitutively express selectins and VCAM-1, which are responsible for rolling and adhesion, whereas BDEC, LDMVEC, and HUVEC do not express these molecules under normal conditions. Therefore, inserts coated with BDEC, LDMVEC, and HUVEC can be pretreated with TNF $\alpha(10 \mathrm{ng} / \mathrm{ml}, 4 \mathrm{hr})$ or other factors to mimic inflammation and induce expression of homing molecules.

\section{Testing crosstalk with the local microenvironment}

There is growing interest in understanding how the local microenvironment regulates the functions of EC and participates in cell extravasation. Our results demonstrate that insertion of a monolayer of stromal cells beneath the EC monolayer significantly increases extravasation of circulating cells. This finding demonstrates that crosstalk between EC and stroma enhances the ability of the EC to support extravasation of circulating cells. Moreover, the insertion of cells from different microenvironments (e.g. mesenchymal stem cells, lung fibroblasts, tumor cells, astrocytes) could help to mimic specific vascular beds. In particular, a combination of brain-derived EC and astrocytes could be a useful approach to mimic the blood-brain barrier. Similarly, cells obtained from patients, or normal cells manipulated in vitro, could help mimic a specific diseased microenvironment.

In our studies, we used lower inserts with stromal cells grown to $50 \%$ confluence. Although the optimal density of microenvironmental cells on the inserts will depend on the goals of the study, this can be readily manipulated and controlled.

\section{Selecting a shear stress rate}

The shear stress of $0.8 \mathrm{dyn} / \mathrm{cm}^{2}$ was used here because this has been demonstrated by Von Andrian et al. to be the shear stress in the bone marrow microvasculature, where hematopoietic progenitor cells exit the circulation and enter tissues under normal physiological conditions ${ }^{15}$. In contrast, higher levels of shear stress are observed in larger vessels, where cell extravasation is limited. Therefore, high shear stress rates could be used as additional controls for experiments examining extravasation of various cell types.

The survival of cells under shear stress depends on several factors, including the cell type and ex vivo cell treatments (Goncharova et al., unpublished observations), and should thus be carefully evaluated during the test. Cell sensitivity to differing levels of shear stress (shear stress resistance) could be evaluated by programming the peristaltic pump to increase the shear stress rate incrementally from $0.8 \mathrm{dyn} / \mathrm{cm}^{2}$ to $6 \mathrm{dyn} /$ $\mathrm{cm}^{2}$. During these tests, cells can be collected periodically from the gas exchange chamber to monitor cell death using assays such as trypan blue exclusion, annexin $\mathrm{V}$ and $\mathrm{PI}$ staining, and apoptosis marker expression.

If experiments are designed to test the shear stress resistance of ex vivo engineered cells or cells derived from parenchyma, it is recommended that additional positive controls, such as blood borne cells, are included in the experiments.

\section{Selection of the insert pore size and ECM coating}

Inserts are available with several pore sizes $(3,5$, and $8 \mu \mathrm{m})$. The choice of pore size will depend on the size and properties of the circulating test cells, which is also the case for static Transwell assays. Inserts with a large pore size $(8 \mu \mathrm{m})$ are recommended to test extravasation of large cells such as tumor cells of epithelial origin. Leukocytes or hematopoietic stem cells are more commonly tested with $5 \mu \mathrm{m}$ pore inserts, and $3 \mu \mathrm{m}$ pore inserts are best reserved for testing extravasation of smaller cells. However, the test cell size alone is not the only important factor and we recommend testing inserts with several pore sizes. 
In our initial studies, we tested various ECM for their ability to support growth of EC on the inserts and to withstand shear stress for $>4 \mathrm{hr}$. The ECM tested included Matrigel, poly-D-lysine, fibronectin, laminin, type I collagen, Hydrogel, Meta-keratin I, II, III, IV, and Extracel. The best results for EC growth were obtained with Extracel, fibronectin, and collagen. However, in our hands, Extracel at $0.8 \mu \mathrm{g} / \mathrm{cm}^{2}$ significantly reduced the transmigration of test cells across the membrane. Therefore we now use fibronectin $\left(5 \mu \mathrm{g} / \mathrm{cm}^{2}\right)$ or collagen $\left(5 \mu \mathrm{g} / \mathrm{cm}^{2}\right)$ for coating of inserts. However, the optimal choice of ECM will probably be influenced by both the type of EC and the transmigratory properties of the test cells. A preliminary experiment should be performed to test the integrity of the EC monolayer grown on selected ECM. For example, place pre-coated inserts with EC monolayers in Petri dishes filled with culture medium, place the dishes on a shaker to create shear stress (low setting), and incubate at $37^{\circ} \mathrm{C}$ and $5 \% \mathrm{CO}_{2}$ for $12 \mathrm{hr}$. The integrity of the $\mathrm{EC}$ after exposure to the shear stress can be evaluated using crystal violet staining.

\section{Selection of optimal test cell concentrations}

The ability of test cells to exit the circulation depends on many characteristics specific to each cell type. To generate statistically significant results, the circulating cell density must be optimized to ensure that a sufficient number of cells migrate into the positive control wells. Therefore, we recommend performing initial tests with a range of cell densities $\left(\right.$ e.g. $10^{3} / \mathrm{ml}$ to $\left.10^{7} / \mathrm{ml}\right)$ to understand the relationship between the number of cells loaded into the system and the number of cells that undergo transmigration.

In addition, the optimal circulating concentration may differ for the same cell type obtained from different sources. For example, CD34 ${ }^{+}$cells are a heterogeneous cell population containing both hematopoietic stem cells and committed lineage-specific progenitors. They can be obtained from bone marrow, mobilized peripheral blood, and umbilical cord blood. The CD34 ${ }^{+}$cells derived from these three sources possess different homing and engrafting abilities ${ }^{16-18}$, so the conditions for testing these cells in the 3D device should be optimized before a full-scale study.

\section{Selection of optimal cell circulation time}

The optimal circulation time should be determined empirically for each cell type. The minimum time required for circulation could be extrapolated from the results of static migration assays, but this may be extended when the cells are subjected to shear stress. Pilot studies testing circulation times between 4 and $96 \mathrm{hr}$ are recommended.

\section{The gas exchange unit}

The main purpose of the gas exchange unit is to maintain the optimal concentration of $\mathrm{CO}_{2}$ in the medium circulating through the 3D device, similar to the settings in standard tissue culture incubators. The gas exchange unit can also be used for adding test cells and compounds and for collecting probes and samples during the test.

\section{Evaluation of test cell numbers}

Circulating cells can be sampled at varying times during the experiment through the gas exchange unit. At the end of experiment, the cells remaining in the circulation could be collected from the outlet. When the 3D device is disassembled at the end of the experiment, the transmigrated cells can be harvested from the lower wells and collected for further analysis. If the input cells are unlabeled, the transmigrated cells can be stained with trypan blue and live/dead cells enumerated microscopically. Alternatively, the input cells could be labeled with one of the many "cell tracker" dyes available for live cell imaging before loading into the 3D device; in this case, the harvested transmigrated cells should be lysed for quantitation of fluorescence.

\section{Selection of the optimal sample size}

To allow statistical analysis, a sample size must be selected that will provide approximately $80 \%$ power to detect the hypothetical difference in the mean percent changes between two groups when tested at a significance level of 0.05 using a two-sided t-test. Based on our experience with bone marrow cells, we use three wells per experimental condition for the 3D device experiments. However, the optimal sample size will vary with the goal of the experiment and should be determined empirically. Multiple regression statistical tests, two-sided t-tests, and analyses of variance can be used to verify statistical relevance of the results.

\section{Selection of chemokines}

As for all transmigration assays, the choice of chemoattractant will be dictated by the properties of the test cells and the goal of the study. SDF-1 is a well established chemoattractant for hematopoietic cells. In our hands, a concentration of $50 \mathrm{ng} / \mathrm{ml}$ SDF-1 was optimal to stimulate extravasation of bone marrow-derived hematopoietic progenitor cells. We also use C3a and bFGF as chemoattractants for mesenchymal stem cells ${ }^{19}$. However, we recommend titrating each chemokine and cross-titrating combinations of chemokines to identify the optimal concentration range before a full-scale study. For certain cell types, a combination of chemotactic factors may be beneficial. If chemokines for specific test cells are unknown or unavailable, conditioned media from stimulated lymphocytes, fibroblasts, and other cells can be used as a source of chemoattractants.

\section{Selection of the readout parameters}

The versatility of the 3D device will expand the type of transmigration experiments that can be performed, and the success of the experiments will depend on thoughtful consideration and design of the readout parameters. As a rule, cells collected from the upper (circulating) compartment and the lower (static) compartment should be tested for viability at the same time as cell counting. Some cells possess low tolerance to the physiological shear stress observed in the microvasculature. In this case, the number of dead cells will be increased in the upper compartment. The number of adherent cells arrested (or trapped) on the EC layer can be evaluated by immunocytochemistry of markers expressed specifically by the test cells. Antibodies specific for CD31 and VWF can be used to detect EC and to allow discrimination between the test cells and EC. In addition, the integrity of the EC monolayer should be monitored at the end of each test.

The types of analyses performed with the collected cells will depend on the investigators' experimental goals, but could include analyzing changes in gene expression by microarrays and qPCR, surface molecule expression by FACS analysis, activation of signaling pathways by 
FACS and western blotting, and factor secretion by ELISA. An enormous array of functional tests are possible on the collected cells in vitro, as evidenced by our own work in which we cultured the transmigrated bone marrow cells to enumerate the hematopoietic progenitor cells (Figure 3). The collected samples could also be tested in a variety of in vivo assays.

One important parameter that can help to predict the behavior of the test cells in vivo is the tendency of some cells to form aggregates under different rates of shear stress. For example, therapeutic cells that undergo aggregate formation in the 3D device might have a greater tendency to form clumps following intravenous administration and cause acute vascular obstruction in vivo (Goncharova et al., unpublished observations). Aggregate formation could be monitored microscopically by sampling the test cells during or after completion of the 3D device experiment.

\section{Disclosures}

Cascade LifeSciences Inc. possesses exclusive rights to patent No. US 7,927,867 B2 "Device for evaluating in vitro cell migration under flow conditions and methods for uses thereof". SKK is an inventor of the 3D flow chamber device technology and a scientific co-founder and shareholder of Cascade LifeSciences Inc.

\section{Acknowledgements}

We thank Chuck Scott and Victor McKnight from the C.B.S. Scientific Company Inc. (www.cbsscientific.com) for engineering assistance and manufacture of the 3D chamber, gas exchange unit, and inserts. This work was supported by the National Institutes of Health (grants R21DK067084 and R43CA141782 to SKK).

\section{References}

1. Daley, G.Q. The promise and perils of stem cell therapeutics. Cell Stem Cell. 10, 740-749 (2012).

2. Khaldoyanidi, S. Directing stem cell homing. Cell Stem Cell. 2, 198-200 (2008).

3. Laird, D.J., von Andrian, U.H., \& Wagers, A.J. Stem cell trafficking in tissue development, growth, and disease. Cell. 132, 612-630 (2008).

4. Lapidot, T. \& Kollet, O. The brain-bone-blood triad: traffic lights for stem-cell homing and mobilization. Hematology Am. Soc. Hematol. Educ. Program. 2010, 1-6 (2010).

5. Sackstein, R. Glycoengineering of HCELL, the human bone marrow homing receptor: sweetly programming cell migration. Ann. Biomed. Eng 40, 766-776 (2012).

6. Smart, N. \& Riley, P.R. The stem cell movement. Circ. Res. 102, 1155-1168 (2008).

7. Khaldoyanidi, S.K., et al. MDA-MB-435 human breast carcinoma cell homo- and heterotypic adhesion under flow conditions is mediated in part by Thomsen-Friedenreich antigen-galectin-3 interactions. J. Biol. Chem. 278, 4127-4134 (2003).

8. Le Devedec, S.E., et al. Two-Photon Intravital Multicolour Imaging to Study Metastatic Behaviour of Cancer Cells in vivo. Methods Mol. Biol. 769, 331-349 (2011).

9. Barakat, A.I. Responsiveness of vascular endothelium to shear stress: potential role of ion channels and cellular cytoskeleton (review). Int. J. Mol. Med. 4, 323-332 (1999).

10. Fisher, A.B., Chien, S., Barakat, A.I. \& Nerem, R.M. Endothelial cellular response to altered shear stress. Am. J. Physiol. Lung Cell Mol. Physiol. 281, L529-533 (2001).

11. Nerem, R.M. Shear force and its effect on cell structure and function. ASGSB Bull. 4, 87-94 (1991).

12. Topper, J.N. \& Gimbrone, M.A., Jr. Blood flow and vascular gene expression: fluid shear stress as a modulator of endothelial phenotype. Mol. Med. Today. 5, 40-46 (1999).

13. Giavazzi, R., Foppolo, M., Dossi, R., \& Remuzzi, A. Rolling and adhesion of human tumor cells on vascular endothelium under physiological flow conditions. J. Clin. Invest. 92, 3038-3044 (1993).

14. Cinamon, G., et al. Novel chemokine functions in lymphocyte migration through vascular endothelium under shear flow. J. Leukoc. Biol. 69, 860-866 (2001).

15. Mazo, I.B., et al. Hematopoietic progenitor cell rolling in bone marrow microvessels: parallel contributions by endothelial selectins and vascular cell adhesion molecule 1. J. Exp. Med. 188, 465-474 (1998).

16. Arber, C., et al. Graft source determines human hematopoietic progenitor distribution pattern within the CD34(+) compartment. Bone Marrow Transplant. 46, 650-658 (2012).

17. Fuji, S., et al. Peripheral blood as a preferable source of stem cells for salvage transplantation in patients with graft failure after cord blood transplantation: a retrospective analysis of the registry data of the Japanese Society for Hematopoietic Cell Transplantation. Biol. Blood Marrow Transplant. 18, 1407-1414 (2012).

18. Haspel, R.L. \& Miller, K.B. Hematopoietic stem cells: source matters. Curr. Stem Cell Res. Ther. 3, $229-236$ (2008).

19. Schraufstatter, I.U., Discipio, R.G., Zhao, M., \& Khaldoyanidi, S.K. C3a and C5a are chemotactic factors for human mesenchymal stem cells, which cause prolonged ERK1/2 phosphorylation. J. Immunol. 182, 3827-3836 (2009). 